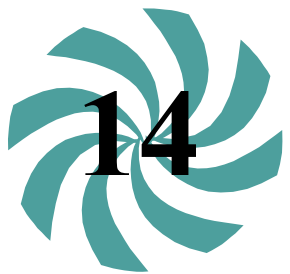

Tecnociencia, Vol. 23, $\mathrm{N}^{\circ} 1: 276-292$

Enero-Junio 2021

\title{
BIOMETRÍA Y DESARROLLO GONADAL DE LA CONCHA NEGRA Anadara tuberculosa EN EL ESTERO CATÉ, GOLFO DE MONTIJO
}

\author{
${ }^{1}$ Annisamyd Del Cid E., ${ }^{1}$ Daniel A. Carneiro G. ${ }^{1}$ Omar Tuñón P., \\ ${ }^{2}$ Orlando Leone, ${ }^{3}$ Italo Goti (iD) \& ${ }^{4}$ Juan A. Gómez H.
}

\begin{abstract}
${ }^{1}$ Universidad de Panamá, Centro Regional Universitario de Veraguas, Escuela de Biología, e-mail anay.03@hotmail.com, omartp05@yahoo.es, carneiro_1031@hotmail.com. 2Departamento de Química Analítica, Universidad de Panamá, Panamá. e-mail orlandoleone@gmail.com. ${ }^{3}$ Universidad de Panamá, Centro Regional Universitario de Azuero, Escuela de Biología, e-mail italo.goti@up.ac.pa ${ }^{4}$ Universidad de Panamá, Escuela de Biología, Departamento de Biología Marina y Limnología, e-mail juanay05@hotmail.com
\end{abstract}

\section{RESUMEN}

Se determinó los aspectos biométricos, y el desarrollo gonadal de la concha negra Anadara tuberculosa en el estero Caté, localizado en el Golfo de Montijo, a los $7^{\circ} 44^{\prime}$ $25^{\prime \prime} \mathrm{N} \mathrm{y} 81^{\circ} 13$ ' 32" O. Se seleccionaron seis estaciones de muestreo, dos de ellas hacia los externos de la desembocadura. En cada sitio durante seis meses se tomaron muestras manualmente, se determinó la morfometría y el peso con ayuda de un vernier y una balanza analítica respectivamente. El desplazamiento modal y la frecuencia de talla fueron estimados con histogramas. Cada gónada fue extraída para determinar el sexo y el grado de madurez, en base a cuatro etapas reproductivas. Se midieron los parámetros fisicoquímicos con una sonda multiparamétrica Marca YSI. A. tuberculosa creció 2,6 mm por mes; la talla máxima registrada fue de 72,25 mm y mínima de 28,05 $\mathrm{mm}$. El mayor peso registrado fue de 126,2 g y el menor de 10,5 g. La proporción de sexos fue de 1,06:1 y el máximo desove se dio en el mes de abril, junto con el aumento de la salinidad en la temporada seca.

\section{PALABRAS CLAVES}

Anadara tuberculosa, bivalvos, gónadas, morfometría, talla, 


\title{
BIOMETRY AND GONADAL DEVELOPMENT OF THE MANGROVE COCKLE Anadara tuberculosa IN THE CATÉ ESTUARY, GULF OF MONTIJO
}

\begin{abstract}
To find out the biometric relationships and gonadal development of the mangrove cockle Anadara tuberculosa, in Cate estuary, Gulf of Montijo, Panama, it was established six sampling stations collected manually each month from February to July 2017, the morphometrics characters were measured with a caliper and the weight with an analytic balance. The physicochemical properties were measured with a YSI multiparameter probe. The monthly growth was calculated by determining the lengthfrequency distribution and the modal analysis. A section of each gonad was extracted to determine the degree of maturity, based on a scale of four reproductive stages. The Sex Ratio was 1.06:1, the maximum spawning was in April coinciding with the maximum increase of salinity in the dry season. A. tuberculosa grew about $2.6 \mathrm{~mm}$ per month, the minimum length was $28.05 \mathrm{~mm}$, and the highest 72.25 , the weight varied between 10.5 to $126.2 \mathrm{~g}$.
\end{abstract}

\section{KEYWORDS}

Anadara tuberculosa, bivalvia, gonads, morphometry, length

\section{INTRODUCCIÓN}

Anadara tuberculosa es una especie de molusco que habita sustratos no consolidados fangosos, limo arcillosos o arcillosos, confinada a las raíces de rodales de Rhizophora mangle y Pelliciera rhizophorae, que reciben el impacto mareal diario, alimentándose mediante materia orgánica (Quizhpe et al., 2017); y se distribuye desde el Golfo de California hasta Tumbes, Perú (Keen, 1971; Campos et al., 1990).

Diversos estudios sobre A. tuberculosa incluyen: edad (Villalobos \& Báez 1983), aspectos reproductivos (Cruz, 1984), estructura poblacional (Campos et al., 1990, Vega, 1998), biométricos (Quijano \& Vega, 2000), tasa de crecimiento (Borda \& Cruz, 2004a), contaminación (Durán et al., 2004), biología general (Rodríguez \& González, 1995 y Jordán \& Gómez, 2006), densidad (Peña, 2017) y rendimiento en carne (Vega \& Robles, 2018). 
La madurez sexual en estos moluscos se alcanza aproximadamente entre 23,2 y 26,2 mm de longitud total (Campos et al., 1990). Posee sexos separados con fecundidad y desarrollo externos. Las gónadas están incluidas en la parte supero-lateral de las vísceras y rodea al intestino y al estómago. En los individuos maduros el tejido gonadal cubre la parte interna distal del pie y la zona dorsal; la expulsión de los gametos ocurre por movimientos continuos de la musculatura gonadal (Cruz, 1984). No existe evidencia de hermafroditismo en esta especie (Cruz \& Jiménez, 1994) y existe pocos datos sobre el desarrollo gonadal en nuestro país.

El presente estudio evalúa las relaciones biométricas, la frecuencia de talla, desplazamiento modal y el desarrollo gonadal de A. tuberculosa en el Estero Caté, Golfo de Montijo.

\section{MATERIALES Y MÉTODOS}

Área de estudio

El Estero Caté está ubicado en el Golfo de Montijo a los $7^{\circ} 44^{\prime} 25^{\prime}$ ' N y $81^{\circ} 13$ ' 32" O, formado por la desembocadura del Río Caté, que vierte sus aguas en dicho golfo, sujeto a cambios estacionales determinado por variaciones durante la temporada de lluvias (Fig.1).

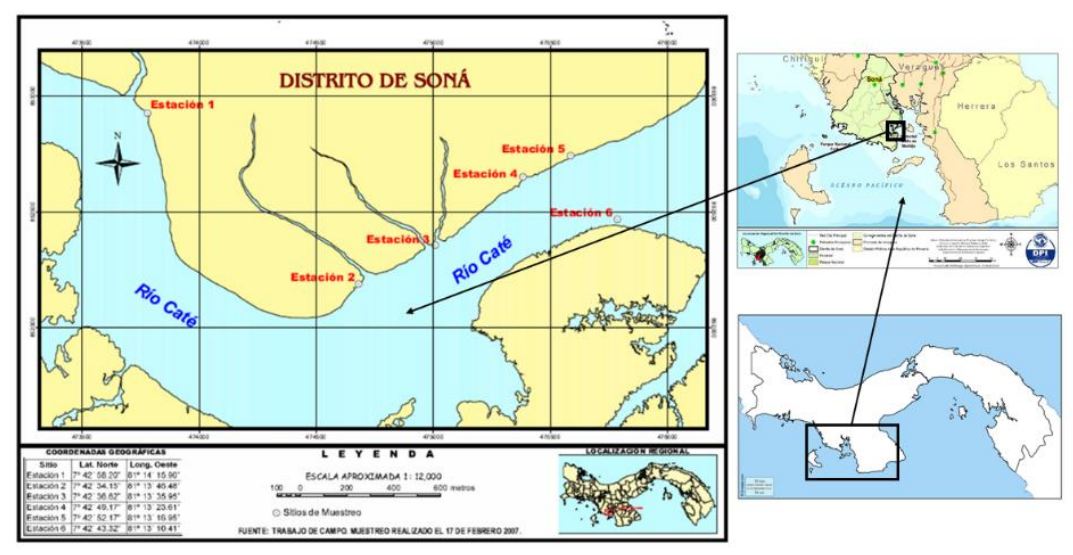

Fig. 1. Mapa del área de estudio 


\section{Metodología}

Se establecieron seis estaciones, cuatro ubicadas a lo largo del estero y dos hacia los puntos externos de la desembocadura (Fig.1); se determinó variaciones espaciales tanto de la talla como de las características reproductivas. Mensualmente se colectaron al azar 30 organismos durante los meses de febrero a julio, a los cuales se midió el largo, ancho y alto, con un calibrador Vernier Modelo Spi 2000 y pesados en una balanza analítica, con precisión de $0,01 \mathrm{~g}$.

El análisis de tallas, a través de histogramas de frecuencia, se utilizó para determinar del crecimiento mediante el método de desplazamiento modal (Petersen, 1892 citado en Pauly, 1983). Se determinó el valor promedio mensual de la talla y peso, de los organismos capturados, a través del período de muestreo y la concordancia ancho y talla mediante la curva de regresión, y el nexo altura - talla y peso - talla.

Doce ejemplares mensuales se utilizaron para los análisis histológicos, de acuerdo a la metodología del Manual de Técnicas Histológicas para Moluscos Bivalvos (Howard et al., 2004). Se hicieron cortes transversales de las gónadas, de 54 micras de espesor, y teñidos en una batería de Hematoxilina-Eosina para la identificación de sexo y madurez de las gónadas, con base a una escala de 4 etapas reproductivas; 1 : Indiferenciado; 2: Desarrollo; 3: Desove; 4: Reabsorción, según Angell (1972) y Gómez y Villalaz (1988). Los registros fisicoquímicos fueron medidos en cada punto de colecta con una sonda multiparamétrica, Marca YSI.

\section{RESULTADOS}

Los promedios de la biometría fueron: talla 53,64 mm, ancho 33,71 mm, altura $37,97 \mathrm{~mm}$, peso completo $48,80 \mathrm{~g}$, peso de la valva $32,47 \mathrm{~g}$ y peso de la gónada de 3,49 g (Cuadro 1). 
Cuadro. 1. Estadística descriptiva $(\mathrm{n}=180)$ para A. tuberculosa en el estero Caté, Golfo de Montijo.

\begin{tabular}{|l|c|c|c|c|}
\hline Parámetro & Promedio & d.s. & Máximo & Mínimo \\
\hline Talla (mm) & 53,64 & 8,22 & 72,25 & 28,05 \\
\hline Ancho (mm) & 33,71 & 7,05 & 48,69 & 18,25 \\
\hline Altura (mm) & 37,97 & 5,59 & 54,07 & 23,94 \\
\hline Peso completo (g) & 48,80 & 24,25 & 126,2 & 10,5 \\
\hline Peso valva(g) & 32,47 & 15,61 & 80,4 & 7,0 \\
\hline Peso de la gónada (g) & 3,49 & 2,64 & 34,4 & 1,4 \\
\hline
\end{tabular}

Los histogramas de estructura de tallas de A. tuberculosa mostraron organismos predominantes entre 50 y $80 \mathrm{~mm}$ en los meses de febrero, marzo. En abril y mayo las tallas fluctuaron entre 35 y $60 \mathrm{~mm}$; a diferencia de junio y julio donde predominaron tallas entre 40 y $75 \mathrm{~mm}$. No hubo registro de tallas menores a $35 \mathrm{~mm}$ a lo largo de los meses de estudio, a excepción del mes de junio cuando se obtuvieron organismos de 25 y $30 \mathrm{~mm}$ (Fig.2).
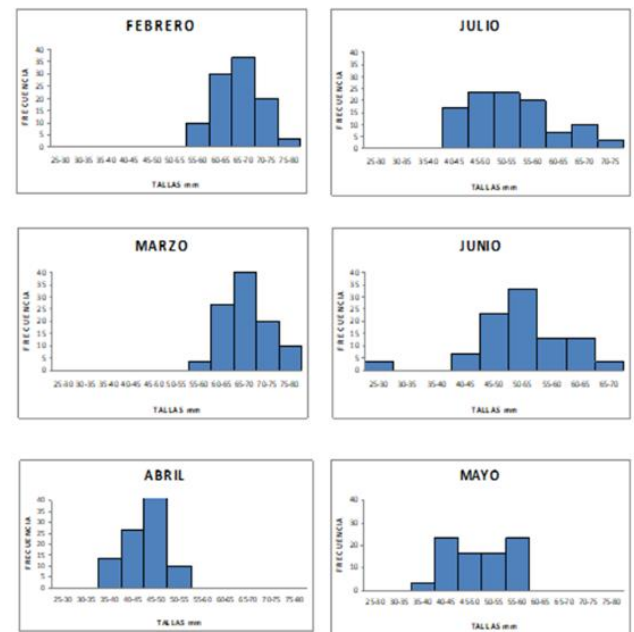

Fig. 2. Histograma mensual de tallas de Anadara tuberculosa en el Estero Caté, Golfo de Montijo. Febrero-Julio 
La moda varió de 58,97 $\mathrm{mm}$ en febrero a 41,06 $\mathrm{mm}$ en junio, sin embargo, el desplazamiento modal indicó crecimiento de abril a mayo: $5,49 \mathrm{~mm}$ y de junio a julio: $10,1 \mathrm{~mm}$, procedentes de varias cohortes

El registro máximo de amplitud de talla fue en junio y el valor extremo de 72,25 mm en julio (Fig. 3), el peso más elevado se obtuvo en julio con 126,2 g (Fig. 4).

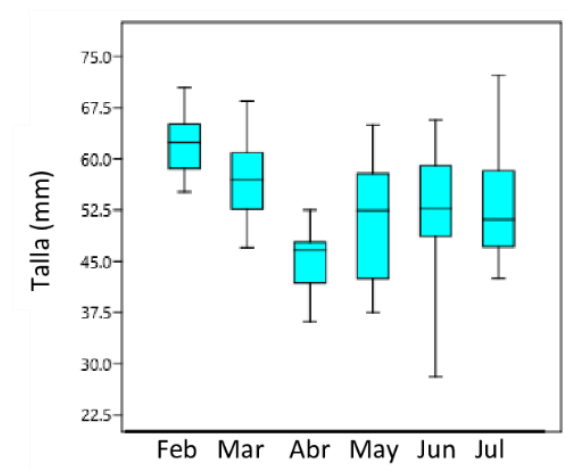

Fig. 3 Talla \pm desviación estándar, durante los meses de muestreo

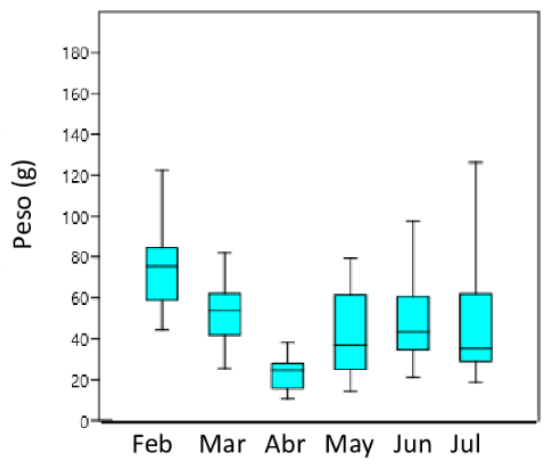

Fig. 4 Peso \pm desviación estándar, durante los meses de muestreo

La relación entre ancho y talla se ajustó mediante la ecuación Ancho = $14,8212+0,6865$ talla, $\mathrm{r}=0,9068$ (Fig. 5). El nexo altura-talla se determinó mediante la ecuación Altura $=17,9504+1,0594$ talla, $\mathrm{r}=$ 0,8662 (Fig. 6), mientras que peso-talla fue: $\mathrm{W}=0,0004 \mathrm{~L}^{2,9334}, \mathrm{R}^{2}=$ 0,8089 p <0,001 (Fig.7). 


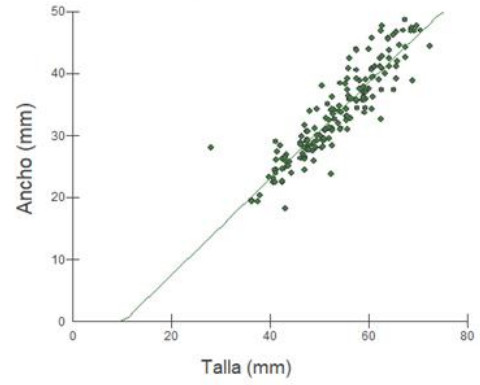

Fig. 5. Ancho vs Talla

de A. tuberculosa de en el Estero Caté

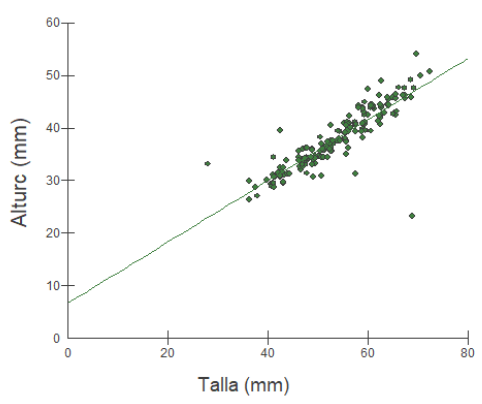

Fig. 6. Altura vs Talla de A. tuberculosa en el Estero Caté

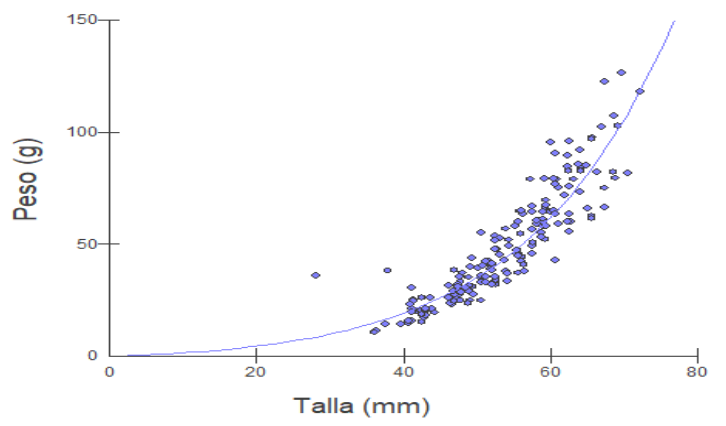

Fig. 7. Peso vs Talla de A. tuberculosa en el Estero Caté

La talla media de primera madurez fue de 53,84 $\mathrm{mm}$ en individuos de ambos sexos, (Fig. 8), para las hembras 54,19 mm y machos 52,01 cm. No existe diferencia significativa de la talla entre hembras y machos $(\mathrm{t}$ $=0,3484 \quad \mathrm{p}=0,7287$ ) 


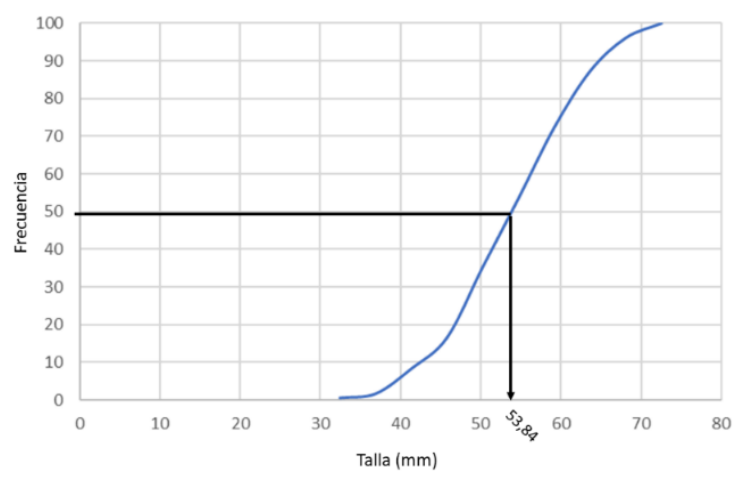

Fig. 8. Talla media de la primera madurez de A. tuberculosa en el Estero Caté.

La proporción sexual fue de 1,06:1, no se encontró diferencia significativa de esta $\left(\mathrm{X}^{2}=0,1343 \mathrm{p}>0,05\right)$.

El análisis histológico y el nexo con la maduración gonadal de mayo y junio presentó la mayor cantidad de individuos en estado indiferenciado con 16,67 \% (Fig. 9), los organismos en período de desarrollo (Fig. 10) registraron un porcentaje máximo en marzo y junio con 41,67\%, la proporción más alta de individuos desovados se observó en abril con $58,33 \%$ y $50 \%$ en julio (Fig. 11). La fase de reabsorción mostró el valor más alto en marzo y mayo con 33,33 \% (Fig. 12). 

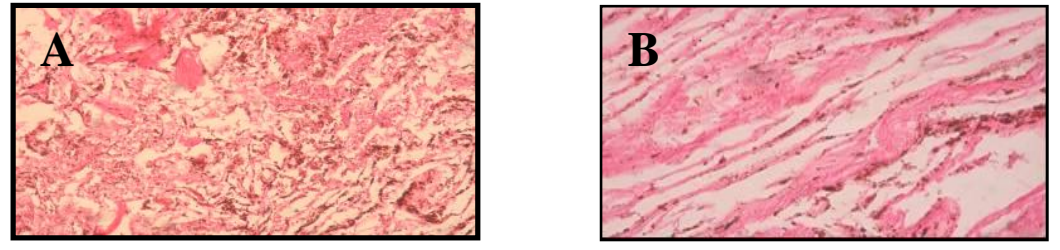

Fig. 9. Etapa 1 (Indiferenciado) de hembras (A) y de machos (B) de A tuberculosa
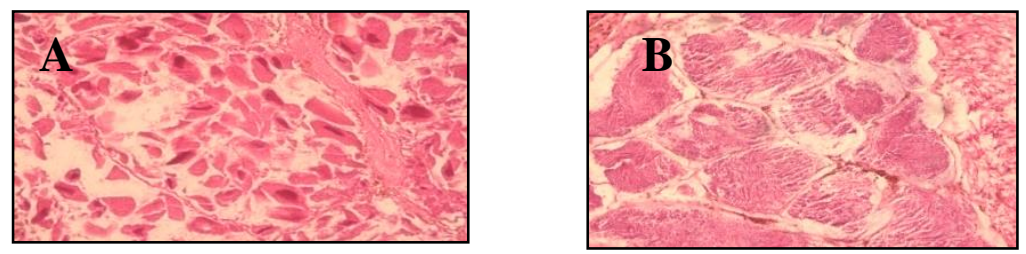

Fig. 10. Etapa 2 (Desarrollo) de hembras (A) y de machos (B) de A. tuberculosa
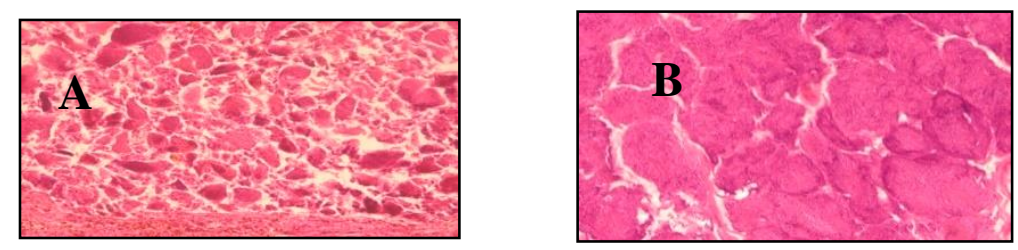

Fig. 11. Etapa 3 (Desove) de hembras (A) y machos (B) de A. tuberculosa
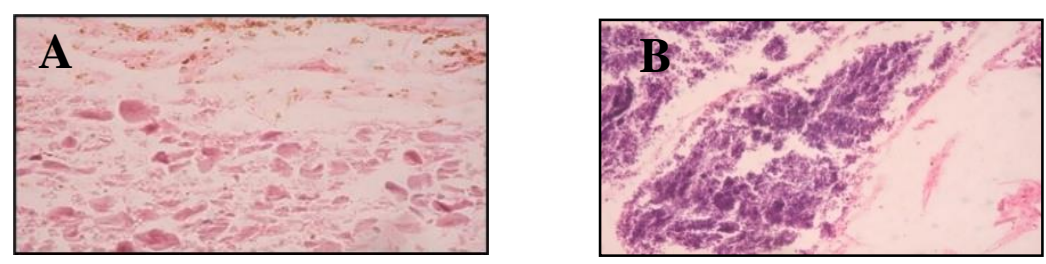

Fig. 12. Etapa 4 (Reabsorción) de hembras (A) y machos (B) de A. tuberculosa

La temperatura del agua promedio fue de $28,58^{\circ} \mathrm{C}$, con ámbito de 27 a $32^{\circ} \mathrm{C}$. La salinidad media fue de 19,58 ups, con niveles bajos en julio, 11,5 ups, mientras que la más alta se obtuvo en abril con 29,6 ups. El $\mathrm{pH}$ fluctuó entre 6,8 y 7,2 con valores extremos en febrero y julio. El oxígeno disuelto fue de 5,24 mg/L, y varió entre 4,12g/L y 6,13 mg/L. 


\section{DISCUSIÓN}

\section{Aspectos Biométricos}

La talla promedio es superior a la encontrada por Jordán y Gómez (2006) en la misma zona de estudio, quienes reportaron variaciones mensuales entre 48,70 y 51,10 mm, en Ecuador, Flores y Licandeo (2010), indican valores de 46,88 $\mathrm{mm}$ para machos y 48,44 $\mathrm{mm}$ en hembras, Cano (2011), en Colombia, informa talla de 44,90 mm, Silva Benavides y Bonilla Carrión (2015) en Costas Rica señalan valores entre 39,52 y 44,94 mm, Ordinola et al. (2020) obtienen talla de 37,3 mm en Perú, mientras que Moreno et al. (2019) con promedios entre 43,42 mm a 47,82 $\mathrm{mm}$ de acuerdo al sitio de colecta.

La variación de las tallas puede ser producto del efecto directo de la captura mensual de los organismos y la presencia de cohortes distintas en los puntos de muestreos. El aumento de tallas máximas en junio puede asociarse al cambio de temporada, donde los aportes de origen continental son generados por escorrentía producto de las lluvias. En ese sentido Cañas y Sierra (2019) indican que estas al dirigirse a la desembocadura de los ríos incrementa la disponibilidad de nutrientes.

El ancho y la altura encontradas en este estudio están dentro de los valores reportados por Palacio (2008), sin embargo, supera los indicados por Cano (2011). El peso promedio total coincide con Jordán y Gómez (2006), pero superior a lo señalado por Cano (2011) y por Silva Benavides y Bonilla Carrión (2015).

\section{Desplazamiento modal}

Los resultados del análisis de desplazamiento modal en abril y junio confirman presencia de varias cohortes, y corresponde parcialmente con lo reportado por Vega (1998) al inicio de la temporada lluviosa y sugiere crecimiento que difiere con lo observado por Villalobos y Báez (1983), quienes señalan que la "piangua" alcanza, en promedio, $20 \mathrm{~mm}$ de longitud en el primer año de vida $(1,8 \mathrm{~mm} / \mathrm{mes}$, aproximadamente), la tasa de crecimiento se reduce a la $\operatorname{mitad}(0,9 \mathrm{~mm} / \mathrm{mes})$ en el segundo año. Estos autores indican que varía de acuerdo a la talla, $0,19 \mathrm{~mm} / \mathrm{mes}$ en individuos de $37,3 \mathrm{~mm}, 0,12 \mathrm{~mm} / \mathrm{mes}$ para organismos de $47,7 \mathrm{~mm}$ y $0,03 \mathrm{~mm} / \mathrm{mes}$ en individuos con longitudes $58 \mathrm{~mm}$. 
Ancho vs talla y altura vs talla

Las relaciones ancho y altura con la talla concuerdan con lo encontrado por Mendoza (2002) y demuestra que conforme aumenta la talla se incrementa proporcionalmente la anchura y la altura del organismo. No obstante, Mendoza y Alvitres (2016) encuentran una relación espesor talla más baja, donde reportan individuos entre 11,36 y 27,77 mm. Según Lucero et al. (2013) el aumento de esta relación es indicativo del bajo nivel de captura del recurso.

Talla media de primera madurez

La talla de primera madurez es superior a la reportada en la literatura. En Costa Rica, valores de 23 y 26,2 mm (Ampie \& Cruz, 1989), en la misma zona se ha observado valores mínimos de 39,5 $\mathrm{mm}$ y 46,68 $\mathrm{mm}$ (Borda \& Cruz 2004b, Lucero et al., 2012 y Lucero et al., 2013). Manjarrés-Villamil et al. (2013) indican que en la Bahía Málaga, Colombia, la talla de primera madurez para $A$. similis es de $42,30 \mathrm{~cm}$, lo que indica el buen estado de los individuos, mientras que para $A$. tuberculosa es de 23 a $26 \mathrm{~mm}$.

Diferenciación sexual y estadio gonadal

A tuberculosa presenta un carácter dioico (sexos separados), lo que concuerda con lo que señalan Ampie y Cruz (1989) y Silva Benavides y Bonilla Carrión (2001). La proporción sexual de hembras para machos en el Estero Caté concuerda con la expresada por algunos investigadores para el Golfo de Montijo (Rodríguez \& González, 1995, Jordán \& Gómez, 2006) y Coiba (Quijano \& Vega, 2000), pero difiere de lo que reporta Mendoza (2002) en los manglares de Puerto Pizarro, Tumbes. Según Alemán et al. (2016) esta proporción es de 1,66:1 y Ordinola et al. (2020), 1,40:1. Recientemente, Díaz (2018) encuentra una relación sexual de 7,89:1 en los manglares de David y 6,23:1 en el Área de Recursos Manejados Humedal Golfo de Montijo.

Los reportes de talla de primera madurez permiten entender el comportamiento sexual en ejemplares de A. tuberculosa; los individuos capturados están por encima de lo reportado por Ampie y Cruz (1989) para la misma especie, quienes indican valores entre 23,2 y 26,2 mm. La madurez sexual, acompañada con el desarrollo de los ovocitos, se 
estudia con mejor precisión a través de cortes histológicos como lo han hecho Maldonado (2006) y García et al. (2008).

A. tuberculosa muestra desoves continuos durante el período de estudio, producto de diferentes cohortes, lo que concuerda con lo encontrado por Quijano y Vega (2000) quienes señalan lo mismo para Isla Coiba, a lo largo del año. Los mayores picos de desove son en febrero y abril, donde la salinidad aumenta, lo que se asemeja a lo expuesto por Borda y Cruz (2004b), en Colombia, a diferencia de lo planteado por Lucero et al. (2013) para la Bahía de Málaga, Colombia.

Con excepción de la salinidad no parece existir ninguna otra coincidencia de las etapas reproductivas con los demás parámetros fisicoquímicos, ya que estos se mantienen estables durante el periodo de estudio. Esta, al igual que la temperatura son factores externos que influyen en el ciclo reproductivo de los bivalvos (Silva Benavides y Bonilla Carrión, 2001; García et al., 2008).

La reabsorción se presenta con mayor abundancia en el mes de junio y menor frecuencia en abril, sin embargo, Tuñón et al. (2020) reportan más individuos en septiembre y abril, en el Estero Farfán, Golfo de Montijo.

La gran estabilidad que tienen los manglares puede haber creado un mecanismo para que las especies del género Anadara, maduren a través de todo el año, lo que muestra una conducta reproductiva marcada.

\section{CONCLUSIÓN}

La talla y el peso promedio encontrados supera a los reportados en otros estudios, igual que la talla de primera madurez, lo cual indica que probablemente los bancos no están sometidos a presión de captura.

La frecuencia de tallas muestra tres cohortes en el período de estudio, enero, abril y julio, reflejado en la variabilidad de la moda. Se confirma la relación lineal, directamente proporcional, del ancho y el alto con 
respecto a la talla y el nexo peso talla resulta ser geométrico e isométrico positivo.

Se definen marcadamente las etapas de desarrollo gonadal y sugieren desoves parciales durante el proceso de maduración de las cohortes.

\section{REFERENCIAS}

Alemán, S., Ordinola, E. y Montero, P. (2016). Concha negra Anadara tuberculos (Sowerby, 1833) y concha huequera Anadara similis (C.B. Adams, 1852) en los manglares de Tumbes, Julio 2016. IMARPE Informe, 46(2), 279-292.

Ampie, C. L. y Cruz, R. A. (1989). Tamaño y madurez Sexual de Anadara tuberculosa (Bivalvia: Arcidae) en Costa Rica. Brenesia, 31, 21-24.

Angell, C. L. (1972). Maduración gonádica y fijación de Cassostrea rhizophorae en una laguna hipersalina del Nororiente de Venezuela. Mem. Soc. Cienc. Nat. La Salle, 32(93), 218-219.

Borda, C. A. y Cruz, R. (2004a). Crecimiento y tasa de mortalidad del bivalvo Anadara tuberculosa (Sowerby, 1833) en el Pacífico colombiano. Rev. Invest. Mar., 25(3), 177-184.

Borda C. y Cruz R. (2004b). Reproducción y reclutamiento del molusco Anadara tuberculosa (Sowerby, 1833) en el Pacífico Colombiano. Rev. Invest. Mar., 25 (3), 185-195.

Campos J. A, Fournier, M. L. y Soto, R. (1990). Estimación de la población de Anadara tuberculosa (Bivalvia: Arcidae) en SirpeTérraba, Costa Rica. Rev. Biol. Trop., 38(2B), 447-480.

Cano, J. L. (2011). Caracterización morfométrica de Anadara tuberculosa y A. similis en la costa pacífica colombiana. [Tesis de Licenciatura, Universidad del Valle, Colombia]. 
Cañas, F. A. y Sierra, S. O. (2019). Análisis bromatológico proximal en Anadara tuberculosa (concha peluda) de la Bahía de Jiquilísco Departamento de Usulután El Salvador. [Tesis Licenciatura, Universidad de El Salvador].

Cruz, R. A. (1984). Algunos Aspectos de la reproducción de Anadara tuberculosa (Pelecypoda: Arcidae) de Punta Morales, Puntarenas, Costa Rica. Rev. Biol. Trop., 32(1), 45-50.

Cruz, R. A. y Jiménez, J. A. (1994). Moluscos asociados a las áreas de manglar de la costa Pacífica de América Central. Edit. Fundación UNA.

Díaz, L. (2018). Análisis de la condición reproductiva en la concha negra Anadara tuberculosa, capturada en los manglares de David y del Área de Recursos Manejados Humedal Golfo de Montijo, pacífico panameño. [Tesis de Maestría Universidad de Panamá].

Durán I. L., Fuentes, M. V. y Gómez, J. A. (2004). Concentración de cadmio, plomo y cobre en Anadara tuberculosa en manglar de isla Taborcillo, Punta Chame, República de Panamá. Tecnociencia, 6(2), 91104.

Flores, L. y Lincadeo, R. (2010). Size composition and sex ratio of Anadara tuberculosa and Anadra similis in a mangrove reserve from the northwest of Ecuador. Rev. Biol. Mar. Oceanogr. 45(3), 541-546.

García, F., Hernández, A., García, A., Villalejo, M. \& Rodríguez, S. (2008). Ciclo reproductivo de Anadara tuberculosa (Sowerby, 1833) (Arcidae) En Bahía Magdalena, México. Rev. Biol. Mar. Ocean., 43(1), 143-152.

Gómez, J. A. y Villalaz, J. R. (1988). Ciclo anual de maduración sexual de la conchuela Argopecten circularis. Scientia. Panamá, 3, 67-77

Howard, D. W., Lewis, E. J., Keller, B. J. y Smith, C. S. (2004). Histological techniques for marine bivalve mollusks and crustaceans. NOAA $2^{\text {nd }} \mathrm{Ed}$. 
Jordán, L. y Gómez, J. A. (2006). Evaluación Biológica de Anadara tuberculosa en el Golfo de Montijo, República de Panamá. Tecnociencia, 8(2), 191-205.

Keen, A. M. (1971). Sea shells of tropical west America. 2nd. Edition. Stanford university Press, Stanford California.

Lucero, C., Cantera, J. y Neira, R. (2012). Pesquerías y crecimiento de la piangua (Arcoida: Arcidae) Anadara tuberculosa en la Bahía de Málaga del Pacífico colombiano, 2005 - 2007. Rev. Biol. Trop., 60(1), 203-217.

Lucero, C., Cantera, J., Gil-Agudelo, D., Muñoz, O., Zapata, L. A., Cortés, N., Gualteros, W. O. y Manjarrés, A. (2013). Análisis temporal de la biología reproductiva y el reclutamiento del molusco bivalvo Anadara tuberculosa en la costa del Pacífico colombiano. Rev. Biol. Mar. Ocenogr., 48(2), 321-334.

Maldonado, J. V. (2006). Aspectos Biológicos y Pesqueros de la concha prieta (Anadara tuberculosa) en el Puerto Morro entre septiembre y diciembre 2005. Instituto Nacional de pesca. Investigación de los Recursos Acuáticos y su Ambiente.

Manjarrés-Villamil, A. E., Lucero-Rincón, C. H., Gualteros, W. O., Cantera-Kintz, J. R. y Gil-Agudelo, D. L. (2013). Abundancia y madurez sexual de Anadara similis en el manglar de Luisico, Bahía Málaga, Pacífico Colombiano. Bol. Invest. Mar. Cost., 42(2), 215-231.

Mendoza, O. (2002). Estructura por Tallas, Densidad Poblacional y Relación Peso Longitud de Anadara tuberculosa en los Manglares de Puerto Pizarro y Zamurilla - Tumbes, Perú. Informe Técnico.

Mendoza, O. M. y Alvitres, V. (2016). Crecimiento y supervivencia de Anadara tuberculosa (Sowerby, 1833) a tres densidades. Revista de Investigación Científica Manglar, 12(1), 55-64.

Moreno, J., Alemán, C. y Bonilla, R. E. (2019). Aspectos biométricos y reproductivos de Anadara tuberculosa (Sowerby, 1833) (Bivalvia: 
Arcidae) en dos sitios de extracción de Esmeraldas y El Oro, Ecuador, durante el último trimestre de 2016. Rev. Mar. Cost., 11(2), 31-43.

Palacio, J. G. (2008). Explicación y conservación de la Piangua: Anadara tuberculosa (Sowerby, 1833) y Anadara similis (C.B. Adams, 1852), en San Andrés de Tumaco. [Tesis de Licenciatura, Universidad de Los Andes].

Pauly, D. (1983). Some simple methods for the assessment of tropical fish stocks. FAO Fish. Tech. Pap., 234, 1-52.

Peña, Y. (2017). Densidad, biometría y rendimiento en carne de la concha negra (Anadara tuberculosa) en el área de Recurso Manejados Humedal Golfo de Montijo, Provincia de Veraguas, Panamá. [Tesis de Licenciatura, Universidad de Panamá].

Ordinola, E., Alemán, S. y Montero, P. (2020). Concha negra Anadara tuberculosa (Sowerby, 1833) y concha huequera Anadara similis en los manglares de Tumbes, Setiembre 2018. Informe IMARPE 47:141-158.

Quijano, C. Y., y Vega, I. M. (2000). Estructura de Talla, Reproducción y Biometría de Anadara tuberculosa (Bivalvia: Arcidae) en el Parque Nacional Coiba. [Tesis de Licenciatura. Universidad de Panamá].

Quizhpe, P. F., Yánez, D. M, y Jimbo, J. I. (2017). Crecimiento y engorde de concha prieta (Anadara tuberculosa) en corrales en los manglares de la isla Payana. Universidad Técnica de Machala. Conference Proceedings Vol. 1, No. 1.

http://investigación.utmachala,edu.ec/proceedings/index.php/utmach

Rodríguez, G. y González, F. (1995). Evaluación de Algunos Aspectos de la Biología de Anadara tuberculosa (Bivalvia: Arcidae) en el Manglar de Diáfara, Mariato-Veraguas, Panamá. [Tesis de Licenciatura. Universidad de Panamá].

Silva Benavides, A. M. y Bonilla Carrión, R. C. (2001). Abundancia y morfometría de Anadara tuberculosa y Anadara similes (Mollusca: 
Bivalvia) en el manglar de Purruja, Golfo Dulce, Costa Rica. Rev. Biol. Trop., 49(2), 315-320.

Silva Benavides, A. M. y Bonilla Carrión, R. C. (2015). Estructura de la población y distribución de Anadara tuberculosa Sowerby (1833) (Mollusca: Bivalvia) en los manglares de Golfito y Playa Blanca de Puerto Jiménez, Golfo Dulce, Costa Rica. Rev. Biol. Trop., 63(Suppl. 1), 287-298.

Tuñón, O., Chang, J. C., Del Cid, A., Goti, I. y Gómez, J. A. (2020). Concentración de metales pesados $(\mathrm{Cu}$ y $\mathrm{Cd})$ en tejido gonadal de $A$. tuberculosa en el estero Farfán, Golfo de Montijo. Tecnociencia, 22(2), 227-243.

Vega, A. J. (1998). Estructura de población, rendimiento y épocas reproductivas de Anadara spp. (Bivalvia: Arcidae) en la reserva Forestal Térraba-Sierpe, Puntarenas, Costa Rica - con recomendaciones para su manejo. [Tesis de Maestría, Universidad de Costa Rica].

Vega, A. y Robles, Y. (2018). Comparación del aprovechamiento del recurso concha negra (Anadara tuberculosa) en los manglares de Montijo y David, Golfo de Chiriquí. Informe Final de Investigación, Universidad de Panamá.

Villalobos, C. R. y Báez, A. L. (1983). Tasa de Crecimiento y Mortalidad en Anadara tuberculosa (Bivalvia: Arcidae) bajo dos Sistemas de Cultivo en el Pacífico de Costa Rica. Rev. Lat. Acui., 17, 154.

Recibido 21 julio 2020, y aceptado 16 noviembre 2020. 